\title{
GLOBAL PROPERTIES OF SPHERICAL CURVES
}

\author{
JOEL L. WEINER
}

Let $\alpha$ be a closed curve regularly embedded in Euclidean three-space satisfying suitable differentiability conditions. In addition, suppose $\alpha$ is nonsingular, i.e., free of multiple points. In 1968, B. Segre [4] proved the following about such curves.

Theorem. If $\alpha$ is nonsingular and lies on a sphere, and 0 denotes any point of the convex hull of $\alpha$ with the condition that 0 (if lying on $\alpha$ ) is not a vertex of $\alpha$, then there are always at least four points of $\alpha$ whose osculating plane at each of those points passes through 0 . If 0 is a vertex of $\alpha$ then there are at least three points of $\alpha$ whose osculating plane at each of those points passes through 0 .

All terms used in the statement of the theorem are defined later in this paper.

To quote H. W. Guggenheimer [2] who reviewed [4], "The 12-page proof is rather complicated." Here we present a shorter and hopefully more transparent proof of this theorem. In addition, we need only require that the spherical curve $\alpha$ be of class $C^{2}$ whereas Segre's proof requires $\alpha$ be of class $C^{3}$. Also, we obtain, with no extra effort, a similar theorem which holds if $\alpha$ 's only singularity is one double point; in this case, the above mentioned minimums must be reduced by two.

In the last section of this paper we characterize spherical curves with the following property: for every point 0 of the convex hull of $\alpha$, other than a vertex of $\alpha$, there exists the same (necessarily even) number of distinct points of $\alpha$ whose osculating plane at each of those points passes through 0 .

The proofs of many results in this paper ultimately depend on ideas contained in a paper by W. Fenchel [1].

Throughout this paper we use the following conventions. By a curve we mean a regular $C^{2}$ function $\alpha: D \rightarrow E^{3}$, where $D$ is an interval (with or without end points) or a circle, and $E^{3}$ is Euclidean three-space. We let $\alpha$ denote both the function and its configuration $\alpha(D)$ in $E^{3}$. When $D$ is a circle we say $\alpha$ is closed. If $D$ is a closed interval we may sometimes refer to $\alpha$ as an arc. We say a point $P$ in $E^{3}$ is a multiple point of $\alpha$ if it is the image of $k>1$ points of $D$. If $k=2$ then $P$ is called a double point. At a multiple point $P$ we will think of $P$ as $k$ distinct points each traversed once by $\alpha$ as we traverse $D$ once. If $\alpha$ has no multiple points, then we say $\alpha$ is nonsingular.

Received July 16, 1975. This research was supported by NSF Grant GP 43030. 


\section{Geodesic curvature}

Let $\alpha$ be an oriented spherical curve; i.e., $\alpha$ lies on a sphere $S$ in $E^{3}$ and has a preferred direction of traversal. Let $S$ be oriented, say, with respect to the outward pointing normal. We denote by $k$ the geodesic curvature of $\alpha$ as a curve in $S$. It is defined by $k=\left(d^{2} \alpha / d s^{2}\right) \cdot n$, where $s$ is the arc length parameter of $\alpha$ consistent with its orientation, and $n$ is $d \alpha / d s$ rotated $+90^{\circ}$ in the tangent plane to $S$ at its point of contact with $S$. Since $\alpha$ is $C^{2}, k$ is a continuous function on $\alpha$.

At each point $P$ of $\alpha$ there is in $S$ a circle tangent to $\alpha$ which best approximates $\alpha$ near $P$. This circle $\omega(P)$ is the osculating circle to $\alpha$ at $P$; it is easy to see that $\omega(P)$ is the intersection of the sphere $S$ and the osculating plane $\pi(P)$ to $\alpha$ at $P$, when $\alpha$ is viewed as a curve in $E^{3}$. We have the following obvious lemma.

Lemma 1. Let $\alpha$ be a spherical curve and $P \in \alpha$. Then $k(P)=0$ if and only if $\pi(P)$ goes through the center of $S$.

We will need some lemmas about spherical curves proved by Fenchel [1]. Actually we state mild generalizations of these lemmas; see [1], [5] for their proofs. In these lemmas we speak of a set on the sphere being to the left of a curve. By this we mean that when the tangent vector to the curve in the preferred direction is rotated $+90^{\circ}$ it points into the set. Also when we say a point $P$ is between points $A$ and $B$ we mean that either $A$ and $B$ are antipodes or if $A$ and $B$ are not antipodes then $P$ lies on the shorter geodesic arc through $A$ and $B$.

Lemma 2. A nonsingular spherical curve $\alpha$ with $k \geq 0$ and not identically zero connects two points $A$ and $B$ of a great circle $\gamma$ without otherwise meeting it. Then $A$ and $B$ are not antipodes of one another. In addition the region bounded by the curve and the smaller great circular arc $A B$ of $\gamma$ and lying in a hemisphere is to the curve's left.

Lemma 3. Let $\alpha$ be a nonsingular spherical curve with $k \geq 0$, and let $\gamma$ be an arbitrary great circle which meets $\alpha$ in at least two points. Then there is a subarc $\alpha_{\gamma}$ of $\alpha$ with the following characteristics:

1. The end points $A$ and $B$ of $\alpha_{\gamma}$ lie on $\gamma$.

2. $\alpha_{\gamma}$ has otherwise no points in common with $\gamma$.

3. All other points of intersection of $\alpha$ with $\gamma$ lie between $A$ and $B$.

Remark. If $\alpha_{\gamma}$ contains a point $P$ for which $k(P)>0$, then $A$ and $B$ are not antipodal by Lemma 2. In particular, more than a half circle of $\gamma$ is free of points of intersection with $\alpha$.

\section{Fenchel's theorem}

The convex hull of a point set $M$ in Euclidean space is the smallest convex set containing $M$. Let $\Omega$ be the convex hull of a spherical curve $\alpha$. The next lemma characterizes the points of $\Omega$; for its proof see [1, Satz A]. 
Lemma 4. For 0 to be an element of $\Omega$ it is necessary and sufficient that there exists a plane $\lambda$ through 0 such that 0 is in the convex hull of $\alpha \cap \lambda$.

Throughout this section we take 0 to be the center of the sphere $S$ on which $\alpha$ lies. With this choice for 0 , Lemmas 3 and 4 lead immediately to a theorem due to Fenchel [1, Satz II']. This theorem is restated to include the possibility that 0 is an element of the boundary of $\Omega$ as well as the interior of $\Omega$.

Theorem 1 (Fenchel). Suppose $\alpha$ is closed and nonsingular except perhaps for one double point. If $0 \in \Omega$, and $\alpha$ does not contain a great semicircular arc, then the geodesic curvature of $\alpha$ changes sign at least twice.

The same lemmas can be used to prove the following extension of Theorem 1. This will be shown here.

Theorem 2. Suppose $\alpha$ is closed and nonsingular. If $0 \in \Omega$, and $\alpha$ does not contain a great semicircular arc, then the geodesic curvature of $\alpha$ changes sign at least four times.

Remark. It is easy to construct examples of closed nonsingular spherical curves whose geodesic curvature changes sign only twice and which necessarily contain a great semicircular arc. It is a consequence of Lemma 2 that these curves lie in a hemisphere determined by the great semicircular arc.

The remainder of this section is devoted to a proof of Theorem 2. Before we proceed we introduce some notation. If $\alpha$ is a non-closed spherical curve, and $P, Q$ are two points of $\alpha$, then by $P \alpha Q$ we mean the oriented arc running along $\alpha$ from $P$ to $Q$. If $P, Q$ are two points of the sphere $S$ which are not antipodal, then $P Q$ denotes the smaller great circular arc through $P$ and $Q$ oriented from $P$ towards $Q$. To denote the larger great circular arc connecting $P$ and $Q$, we write $P A Q$ where $A$ is on the great circle through $P$ and $Q$ but $A \notin P Q$. By a Jordan curve we mean a nonsingular continuous image of a circle.

Proof of Theorem 2. Let $\alpha$ be a closed nonsingular curve lying on a sphere $S$ with center 0 , and suppose that $\alpha$ contains no great semicircular arc. In particular, $\alpha$ 's geodesic curvature $k$ is not identically zero. Also suppose $0 \in \Omega$, the convex hull of $\alpha$. By Theorem 1 we already know that $k$ changes sign at least twice. We will show that the supposition that $k$ changes sign only twice leads to a contradiction. Therefore suppose $k$ changes sign twice at the points $A$ and $B$ of $\alpha$. Let $\alpha^{1}$ and $\alpha^{2}$ be the two curves into which $\alpha$ is separated by $A$ and $B$, both oriented so that their geodesic curvature is nonnegative (and, of course, not identically zero). Suppose $\alpha^{1}$ and $\alpha^{2}$ begin at $A$ and end at $B$.

By Lemma 2 there is a plane $\lambda$ through 0 such that 0 is in the convex hull of $\lambda \cap \alpha$. Let $\gamma=\lambda \cap S$; it is, of course, a great circle. There are two cases to consider. Either

1. $\alpha$ meets $\gamma$ in at least three points and these points do not lie in an open half circle of $\gamma$, or

2. $\alpha$ meets $\gamma$ in two points, which are necessarily antipodal. 
Case 1. Let $C, D, E$ be distinct points at which $\alpha=\alpha^{1} \cup \alpha^{2}$ meets $\gamma$ and which do not lie in an open half circle of $\gamma$. We may suppose that $C$ and $D$ are points of $\alpha^{1}$; in fact, suppose $C$ precedes $D$ in $\alpha^{1}$. Since $\alpha^{1}$ meets $\gamma$ in at least two points, Lemma 3 implies that there exists a subarc $\alpha_{\gamma}^{1}$ with the characteristics 1,2 , and 3 of that lemma. Also $\alpha_{\gamma}^{1}$ is not a great semicircular arc. The remark following Lemma 3 implies that $E$ must be a point of $\alpha^{2}$. We may assume that $C$ and $D$ are the end points of $\alpha_{r}^{1}$; if the new $C, D, E$ lie in an open half circle of $\gamma$ so do the old $C, D, E$.

Let $H$ be the closed hemisphere determined by $\gamma$ and not containing $\alpha_{\gamma}^{1}$ except for the end points $C$ and $D$. Let $L$ be the region to the left of the oriented Jordan curve $C \alpha^{1} D \cup D C$ together with its boundary. Lemma 3 implies that $\alpha^{1} \subset H \cup L$. In particular $A, B \in H \cup L$; hence $\alpha^{2}$ must begin and end in $H \cup L$. The boundary of $H \cup L$ is the Jordan curve $\alpha_{r}^{1} \cup D E C$. Now if $\alpha^{2}$ is not contained in $H \cup L$, it must cross the boundary along $D E C$ (excluding the end points $D$ and $C$ ). Remember that $\alpha^{1}$ and $\alpha^{2}$ meet only at $A$ and $B$. We assume without loss of generality that $\alpha^{2}$ crosses $D E C$. If $\alpha^{2} \operatorname{did}$ not cross $D E C$, then it would be tangent to $\gamma$ at $E$. We could then rotate $\lambda$ a bit about the diameter of $S$ through $C$ or $D$ so that $\alpha$ crosses $\gamma$ at points which we still call $C, D, E$ and which still do not lie in an open half circle of $\gamma$. Since $\alpha^{2}$ meets $\gamma$ at least twice, Lemma 3 implies the existence of a subarc $\alpha_{\gamma}^{2}$. Let $\alpha_{r}^{2}$ begin at $F$ and terminate at $G$. Characteristic 3 of $\alpha_{r}^{2}$ implies that at least one of the points $F$ and $G$ is not between $C$ and $D$. At this stage of the argument we suppose that $F$ does not lie between $C$ and $D$. The argument is similar if we suppose that $G$ does not lie between $C$ and $D$.

Consider the oriented Jordan curve $A \alpha^{1} D \cup D F \cup F \alpha^{2} A$. If $D$ and $F$ are antipodal, then here $D F$ is the half great circle not containing $G$; see Fig. 1 .

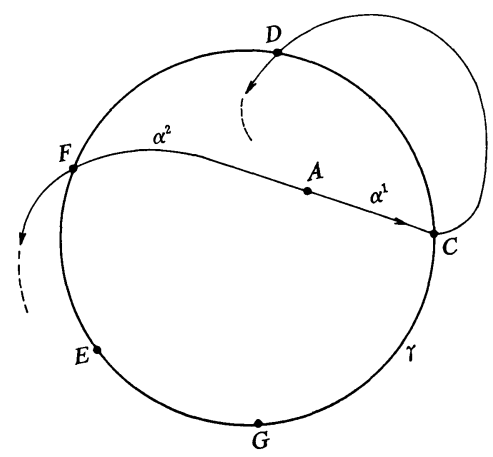

Fig. 1

Note that $D \alpha^{1} B$ and $F \alpha^{2} B$ cannot cross the Jordan curve. That $F \alpha^{2} B$ does not cross $D F$ is the only part of the preceding statement which may not be im- 
mediately clear. However $F \alpha^{2} B$ may only cross $\gamma$ along $F G$ which is less than a half circle; also $D F$ is at most a half circle. Thus $D F$ meets $F G$ only at $F$. Thus $F \alpha^{2} B$ meets $D F$ only at $F$. Now $D \alpha^{1} B$ and $F \alpha^{2} B$ are on opposite sides of the Jordan curve near $D$ and $F$, respectively. This is clear since $\alpha^{1}$ is entering $H$ at $D$ and $\alpha^{2}$ is leaving $H$ at $F$. Thus $B$ is both to the right and the left of the Jordan curve, which is a contradiction.

Case 2. Let $C$ and $D$ be the two points in which $\alpha$ meets $\gamma$. As already noted $C$ and $D$ are necessarily antipodal. This case can be reduced to Case 1 since there must be a great circle through $C$ and $D$ which intersects $\alpha$ at a third point $E$. Clearly $C, D, E$ do not lie in an open half circle.

Remark. We do not use the fact that $\alpha^{1}$ and $\alpha^{2}$ join at $A$ and $B$ in a $C^{2}$ fashion, but only that they begin and end at $A$ and $B$, respectively.

\section{Segre's theorem}

Generally, if $P$ is a point of a curve $\alpha$ then at $P \alpha$ passes through the osculating plane to $\alpha$ at $P$. However if this does not happen we call $P$ a vertex of $\alpha$. Thus by a vertex of a curve $\alpha$ we mean a point $P$ of $\alpha$ with the property that near $P \alpha$ lies on one side of the osculating plane to $\alpha$ at $P$.

Theorem 3. Let $\alpha$ be a closed curve on the sphere $S$ and let $0 \in \Omega, \alpha$ 's convex hull. Then

(i) if $\alpha$ is nonsingular and 0 is not a vertex of $\alpha$, there exist at least four points of $\alpha$ whose osculating plane at each of those points passes through 0 ,

(ii) if $\alpha$ is nonsingular and 0 is a vertex of $\alpha$, there exist at least three points of $\alpha$ whose osculating plane at each of those points passes through 0 ,

(iii) if $\alpha$ 's only singularity is one double point and 0 is not a vertex of $\alpha$, there exist at least two points of $\alpha$ whose osculating plane at each of those points passes through 0 .

The idea behind the proof lies in the observation that Theorem 3 follows trivially from Theorems 1 and 2 by means of Lemma 1 if 0 is the center of $S$. So if 0 is not the center of $S$ we let $\alpha^{*}$ be the projection of $\alpha$ into a sphere $\Sigma$ centered at 0 and apply Theorems 1 and 2 to $\alpha^{*}$ to get the required number of points of $\alpha^{*}$ whose osculating plane at each of those points passes through 0 . If $0 \in \alpha$, then $\alpha^{*}$ is not a closed curve but one can still show that $\alpha^{*}$ has the required number of points whose osculating plane at each of those points passes through 0 . Finally we observe by Lemma 5 that an osculating plane at a point of $\alpha^{*}$ passes through 0 if and only if the osculating plane at the corresponding point of $\alpha$ does so.

We now introduce the notation which will be used in the proofs of Lemma 5 and Theorem 3. Let $\alpha$ be a closed curve on $S$, and $\Omega$ the convex hull of $\alpha$. Suppose that 0 is any element of $\Omega$ and $\Sigma$ is a sphere centered at 0 . Let $p: S$ $\rightarrow \Sigma$ be the projection of $S$ into $\Sigma$ through 0 . When $0 \in \alpha, p$ is understood to be defined only on $S-\{0\}$. Denote the image of $P \in S$ under $p: S \rightarrow \Sigma$ by $P^{*}$. 
If 0 is in interior of $S$, we let $\alpha^{*}$ denote the image of $\alpha$ under $p$. If $0 \in \alpha$, note first that $p(\alpha)$ is contained in a hemisphere $H$ with boundary $\gamma^{*}$, where $\gamma^{*}$ is the intersection of the tangent plane to $S$ at 0 with $\Sigma$. Assume 0 is not a multiple point of $\alpha$; then the limits of $P^{*}$ as $P$ approaches 0 along $\alpha$ first from one side and then the other are two antipodal points on $\gamma^{*}$. We adjoin these points to $p(\alpha)$ and denote the resulting arc by $\alpha^{*}$. When 0 is a multiple point of $\alpha$, we adjoin points of $\gamma^{*}$ to $p(\alpha)$ as above to get a collection of arcs denoted by $\alpha^{*}$. Then let $\Omega^{*}$ be the convex hull of $\alpha^{*}$. Let $\pi(P)$ and $\pi^{*}\left(P^{*}\right)$ denote the osculating planes to $\alpha$ at $P$ and $\alpha^{*}$ at $P^{*}$, respectively.

Lemma 5. Suppose $P \neq 0$. Then $\pi(P)$ passes through 0 if and only if $\pi^{*}\left(P^{*}\right)$ goes through 0 . Moreover, if $\pi(P)$ passes through 0 , then $P$ is a vertex of $\alpha$ if and only if $P^{*}$ is a vertex of $\alpha^{*}$.

Proof. The projection $p: S \rightarrow \Sigma$ is a $C^{\infty}$ diffeomorphism of $S$ onto its image. Thus the order of contact between two curves on $S$ and their images under $p$ on $\Sigma$ is preserved (except if the contact is at $0 \in \alpha$ ).

Let $\omega(P)$ and $\omega^{*}\left(P^{*}\right)$ denote the osculating circles to $\alpha$ at $P$ and $\alpha^{*}$ at $P^{*}$, respectively. Suppose $\pi(P)$ passes through 0 . Since $\omega(P)$ lies in $\pi(P)$ which passes through 0 , its image under $p$ is a (great) circle on $\Sigma$ if $0 \notin \alpha$ and is a half (great) circle on $\Sigma$ if $0 \in \alpha$. Let $\omega(P)^{*}$ denote the circle in which $p(\omega(P))$ lies on $\Sigma$. Since the order of contact is preserved, $\omega(P)^{*}=\omega^{*}\left(P^{*}\right)$. Thus both $\pi(P)$ and $\pi^{*}\left(P^{*}\right)$ contain $\omega(P)^{*}$. Hence $\pi(P)=\pi^{*}\left(P^{*}\right)$ passes through 0 . The converse is proved in an identifical fashion.

Now suppose $\pi(P)$ passes through 0 . Then, by the above, $\pi(P)=\pi^{*}\left(P^{*}\right)$. If $\alpha$ lies on one side of $\pi(P)$ near $P$, clearly $\alpha^{*}$ lies on one side of $\pi^{*}\left(P^{*}\right)$ near $P^{*}$ and conversely. That is, $P$ is a vertex of $\alpha$ if and only if $P^{*}$ is a vertex of $\alpha^{*}$.

Proof of Theorem 3. We separate the proof into two cases according as $0 \in \alpha$ or not.

Suppose $0 \notin \alpha$. Then it is clear that $0 \in \Omega^{*}$ since $0 \in \Omega$. Thus we may apply Theorems 1 and 2 to $\alpha^{*}$ lying on $\Sigma$. If $\alpha$ is nonsingular, so is $\alpha^{*}$; thus $\alpha^{*}$ has at least four points where its geodesic curvature is zero. If $\alpha$ has just one double point, so does $\alpha^{*}$; thus $\alpha^{*}$ has at least two points where its geodesic curvature is zero. By Lemma 1 , at each of these points of $\alpha^{*}$ the osculating plane passes through 0 . Hence by Lemma 5 the osculating planes at the corresponding points of $\alpha$ pass through 0 . Thus we have proved (i) and (iii) for the case $0 \notin \alpha$.

Suppose $0 \in \alpha$ and 0 is not a multiple point of $\alpha$. Assume now $\alpha$ is oriented. By means of $p$ we orient $\alpha^{*}$. Denote the beginning of $\alpha^{*}$ by $A$ and the end by $B$. Let $\omega$ be the osculating circle to $\alpha$ at 0 . Its image under $p$ including end points, denoted by $\omega^{*}$, is a half great circular arc of $\Sigma$. It is easy to see that $\omega^{*}$ also begins at $A$ and ends at $B$. Also $\omega^{*}$ and $\alpha^{*}$ are tangent at $A$ and $B$. If 0 is not a vertex of $\alpha$, then $\alpha^{*}$ is on opposite sides of $\omega^{*}$ in $H$ near $A$ and $B$; see Fig. 2. If 0 is a vertex of $\alpha$, then $\alpha^{*}$ is on the same side of $\omega^{*}$ in $H$ 


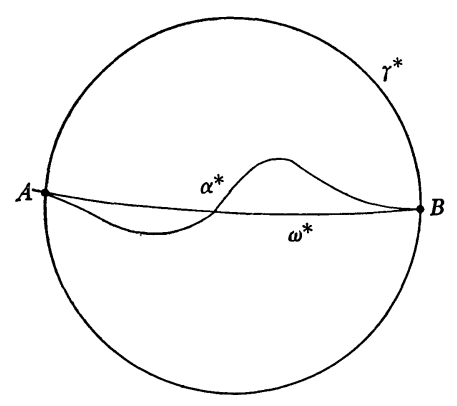

Fig. 2

near $A$ and $B$. Let $k^{*}$ be the geodesic curvature of $\alpha^{*}$. Then using Lemma 2 and the idea of parity, one can show the following hold:

1. $k^{*}$ changes sign at least twice if 0 is not a vertex of $\alpha$ and $\alpha$ is nonsingular,

2. $k^{*}$ changes sign at least twice if 0 is a vertex of $\alpha$ and $\alpha$ is nonsingular,

3. $k^{*}$ changes sign at least once if 0 is not a vertex of $\alpha$ and $\alpha$ 's only singularity is one double point.

Again apply Lemmas 1 and 5, in that order, to prove (i), (ii), and (iii) for the case where $0 \in \alpha$ and 0 not a multiple point of $\alpha$. If 0 is the double point of $\alpha$ the proof of (iii) is immediate.

Corollary. Let $\alpha$ be a $C^{3}$ closed nonplanar curve in $E^{3}$ with no pair of directly parallel tangents. Then $\alpha$ has at least four vertices.

For the proof of this corollary see Segre [4, p. 263] where the same result is proven for $C^{4}$ curves. Our results allow his proof to go through for $C^{3}$ curves. Actually the corollary follows immediately from Theorem 2 and the remark following Theoerm 2 since the tangent indicatrix of a nonplanar curve cannot lie in a hemisphere.

\section{A characterization}

In this section we find a characterization for a (possibly singular) closed curve $\alpha$ lying on the sphere $S$ and having the property that for each point 0 in its convex hull $\Omega$ except for vertices of $\alpha$ there exists the same (necessarily even) number of distinct points of $\alpha$ whose osculating plane at each of those points passes through 0 .

The next lemma is especially important in this section. It follows by means of stereographic projection from a similar fact for plane curves due to Kneser; see [3, p. 48] for Kneser's theorem and its proof. When we say that the circle $\omega$ lies between the (disjoint) circles $\omega^{1}$ and $\omega^{2}$ on the sphere $S$ we mean that $\omega$ is in the connected component of $S-\left(\omega^{1} \cup \omega^{2}\right)$ whose boundary is $\omega^{1} \cup \omega^{2}$.

Lemma 6. Let $\alpha$ be spherical arc with monotone geodesic curvature $k$. Let $P, Q$, and $R$ be three points of $\alpha$ with $Q$ between $P$ and $R$. Then $\omega(Q)$ is be- 
tween $\omega(P)$ and $\omega(R)$ if it is not equal to $\omega(P)$ or $\omega(R)$. Moreover, $\omega(Q)=\omega(P)$ (respectively, $\omega(R)$ ) only if $k(Q)=k(P)$ (respectively, $k(R)$ ).

At this point we make some additional assumptions about the closed spherical curve $\alpha$ which will hold throughout the remainder of this section. First, we require that there exists at most a finite number of points of $\alpha$ at which the geodesic curvature $k$ takes on an extreme value. This is equivalent to requiring that $\alpha$ has at most a finite number of vertices since the vertices of $\alpha$ occur at the extremes of $k$. Secondly, we assume $k$ is strictly monotone between the vertices of $\alpha$. This second condition rules out the possibility of $\alpha$ having an arc of points with the same osculating plane.

Let $B$ denote the closed ball whose boundary $S$ contains the closed curve $\alpha$. Clearly $\Omega \subset B$.

Theorem 4. Suppose $\alpha$ has $n$ vertices. If $0 \in B$, then there exist at most $n$ points of $\alpha$ whose osculating plane at each of those points passes through 0.

Proof. Let $V_{1}, V_{2}, \cdots, V_{n}$ denote the vertices of $\alpha$ as they occur in making one circuit of $\alpha$. Using the notation of $\S 2$, we set $\alpha^{i}=V_{i} \alpha V_{i+1}$ for $i=1$, $2, \cdots, n$, where $V_{n+1}=V_{1}$. We will show for each integer $i$, where $1 \leq i \leq n$, there exists at most one point $P \in \alpha^{i}$ such that $0 \in \pi(P)$. This immediately implies the theorem.

Suppose, to the contrary, that $\alpha^{i}$ contains two points $P$ and $Q$ such that $0 \in \pi(P) \cap \pi(Q)$. In particular, $\pi(P) \cap \pi(Q) \neq \emptyset$; hence $\omega(P) \cap \omega(Q) \neq \emptyset$. This is impossible by Lemma 6 since $k$ is strictly monotone on $\alpha^{i}$.

Remark. Note that $V_{i} \in \alpha^{i-1} \cap \alpha^{i}$ for $i=1,2, \cdots, n$, where $\alpha^{0}=\alpha^{n}$. Hence if $0 \in B$ and, in addition, $0 \in \pi\left(V_{i}\right)$, then there exist strictly less than $n$ points of $\alpha$ whose osculating plane at each of those points passes through 0 .

Corollary. Suppose $\alpha$ has $n$ vertices. If $0 \in \Omega$, then there exist at most $n$ points of $\alpha$ whose osculating plane at each of those points passes through 0.

Let $V_{1}, V_{2}, \cdots, V_{n}$ be the vertices of $\alpha$. Note that $n$ is necessarily even since it is the number of extreme points of the geodesic curvature of $\alpha$.

Theorem 5. Suppose $\omega\left(V_{i}\right) \cap \alpha=\left\{V_{i}\right\}$ for $i=1,2, \cdots, n$. Then for every $0 \in \Omega-\left\{V_{1}, V_{2}, \cdots, V_{n}\right\}$ there exist exactly $n$ points $P_{1}, P_{2}, \cdots, P_{n}$ of $\alpha$ such that $0 \in \pi\left(P_{i}\right)$ for $i=1,2, \cdots, n$, and conversely.

Proof. Let $B^{\prime}=B-\bigcup_{i=1}^{n} \pi\left(V_{i}\right)$. Also let $B_{m}^{\prime}$ be the set of points 0 in $B^{\prime}$ with the property that there exist exactly $m$ points $P_{1}, P_{2}, \cdots, P_{m}$ of $\alpha$ such that $0 \in \pi\left(P_{i}\right)$ for $i=1,2, \cdots, m$.

Let $\Omega^{\prime}=\Omega-\left\{V_{1}, V_{2}, \cdots, V_{n}\right\}$. For $i=1,2, \cdots, n$, the assumption $\omega\left(V_{i}\right)$ $\cap \alpha=\left\{V_{i}\right\}$ implies $\Omega \cap \pi\left(V_{i}\right)=\left\{V_{i}\right\}$. Thus $\Omega^{\prime}$ is a connected subset of $\boldsymbol{B}^{\prime}$. The theorem is proved by showing that for any nonnegative integer $m, B_{m}^{\prime}$ is an open and closed subset of $B^{\prime}$. This implies $\Omega^{\prime} \subset B_{m}^{\prime}$ for some nonnegative integer $m$. Then we show $m=n$.

The fact that $B_{m}^{\prime}$ is both open and closed in $B^{\prime}$ follows in three steps :

Step 1. $B_{m}^{\prime} \subset$ interior $\bigcup_{m \leq j} B_{j}^{\prime}$. Let $0 \in B^{\prime}$ and suppose there exist $m$ points $P_{1}, P_{2}, \cdots, P_{m}$ of $\alpha$ such that $0 \in \pi\left(P_{i}\right)$ and $P_{i}$ is not a vertex of $\alpha$ for 
$i=1,2, \cdots, m$. We will show for each integer $i$, where $1 \leq i \leq m$, there exists a neighborhood $N_{i}$ of $P_{i}$ in $\alpha$ with the property that $U_{i}=\bigcup_{P \in N_{i}} \pi(P)$ $\cap B^{\prime}$ is an open set of $B^{\prime}$ containing 0 . Moreover, we may assume $N_{1}, N_{2}, \cdots$, $N_{m}$ are mutually disjoint. It is then clear that $U=\bigcap_{i=1}^{m} U_{i}$ is a neighborhood of 0 in $\cup_{m \leq j} B_{j}^{\prime}$.

Consider the point $P_{i}$. Since $P_{i}$ is not a vertex there exists an open neighborhood $N_{i}$ of $P_{i}$ in $\alpha$ on which $k$ is strictly monotone. By Lemma $6, N_{i}$ does not contain $P_{j}$, where $j \neq i$. Let $P_{i}^{\prime}$ and $P_{i}^{\prime \prime}$ be the boundary points of $N_{i}$. It follows from Lemma 6 that $\cup_{P \in N_{i}} \omega(P)$ is an open set of $S$; it is the component of $S-\left[\omega\left(P_{i}^{\prime}\right) \cup \omega\left(P_{i}^{\prime \prime}\right)\right]$ containing $P_{i}$. Then $U_{i}=\cup_{P \in N_{i}} \pi(P) \cap B^{\prime}$ is an open set of $B^{\prime}$. In fact $U_{i}$ is the component of $B^{\prime}-\left[\pi\left(P_{i}^{\prime}\right) \cup \pi\left(P_{i}^{\prime \prime}\right)\right]$ containing $P_{i}$. Clearly $0 \in U_{i}$ since $P_{i} \in N_{i}$.

Step 2. $\quad B_{m}^{\prime}$ is closed in $B^{\prime}$. Let $0_{i}, i=1,2, \ldots$, be a sequence of points in $B_{m}^{\prime}$ approaching $0 \in B^{\prime}$. Thus for each $i=1,2, \ldots$, there exist exactly $m$ points $P_{i 1}, P_{i 2}, \cdots, P_{i m}$ of $\alpha$ such that $0_{i} \in \pi\left(P_{i j}\right)$ for $j=1,2, \cdots, m$. By taking subsequences if necessary, we may assume that $P_{i j}$ approaches a point $P_{j}$ as $i$ approaches infinity for $j=1,2, \cdots, m$. By continuity $0 \in \pi\left(P_{j}\right)$ for $j=1,2$, $\cdots, m$. Thus there are at least $m$ points of $\alpha$ whose osculating plane at each of those points passes through 0 unless $P_{j}=P_{k}$ for some $j \neq k$. Suppose this ; then in any neighborhood of $P_{j}=P_{k}$ there exist the distinct points $P_{i j}, P_{i k}$, for $i$ sufficiently large. Since $0_{i} \in \pi\left(P_{i j}\right) \cap \pi\left(P_{i k}\right), \omega\left(P_{i j}\right) \cap \omega\left(P_{i k}\right) \neq \emptyset$. By Lemma $6, P_{j}=P_{k}$ is a vertex of $\alpha$. But this contradicts the assumption $0 \notin \bigcup_{i=1}^{n} \pi\left(V_{i}\right)$. Thus $P_{j} \neq P_{k}$ for all $j \neq k$ between 1 and $m$ inclusive. By Step 1 there exist at most $m$ points $P_{1}, P_{2}, \cdots, P_{m}$ of $\alpha$ with $0 \in \pi\left(P_{j}\right)$.

Step 3. $B_{m}^{\prime}$ is open in $B^{\prime}$. This step follows immediately from Step 1 and Step 2 since $B_{m}^{\prime}=\emptyset$ for $m>n$ by Theorem 4 .

We now know that $\Omega^{\prime} \subset B_{m}^{\prime}$ where $m \leq n$. Suppose $m<n$. We will show this leads to a contradiction. Let $0 \in \alpha \cap \Omega^{\prime}$. Since $0 \in \Omega^{\prime}$, there exist $m$ points $P_{1}, P_{2}, \cdots, P_{m}$ with $0 \in \pi\left(P_{i}\right)$ for $i=1,2, \cdots, m$. In the notation of the proof of Theorem 4 , there exists an arc $\alpha^{i}$ for some integer between 1 and $n$ inclusive with the following property : there exists no point $Q \in \alpha^{i}$ such that $0 \in \pi(Q)$. Thus $\omega\left(V_{i}\right)$ and $\omega\left(V_{i+1}\right)$ do not have 0 between them. Hence, say, $\omega\left(V_{i}\right)$ and 0 are separated by $\omega\left(V_{i+1}\right)$. In particular $V_{i}$ and 0 are on opposite sides of $\omega\left(V_{i+1}\right)$. Thus $\alpha$ must meet $\omega\left(V_{i+1}\right)$ at points other than $V_{i+1}$.

The converse follows from the remark following the proof of Theorem 4.

q.e.d.

It may still be that for every point 0 of $\Omega^{\prime}$ there exists the same number of points of $\alpha$ whose osculating plane at each of those points passes through 0 even though $\omega\left(V_{i}\right) \cap \alpha \neq\left\{V_{i}\right\}$ for some integer $i, 1 \leq i \leq n$. For this to happen the following must be true: if, say, $V_{1}$ is a vertex of $\alpha$ and $\omega\left(V_{1}\right)$ intersects $\alpha$ in more than $V_{1}$, then there must be another vertex $V_{i}$ for some integer $i, 2 \leq i \leq n$, such that $\pi\left(V_{i}\right)=\pi\left(V_{1}\right)$. Also, for points $P$ near $V_{1}$ and $Q$ near $V_{i}, \pi(P)$ and $\pi(Q)$ must be on opposite sides of $\pi\left(V_{1}\right)=\pi\left(V_{i}\right)$. 


\section{References}

[1] W. Fenchel, Über Krümmung und Windung geschlossener Raumkurven, Math. Ann. 101 (1929) 238-252.

[2] H. W. Guggenheimer, Rev. \#4787, Math. Rev. 39 (1970) 871.

[3] - Differential geometry, McGraw-Hill, New York, 1963.

[4] B. Segre, Alcune proprietà differenziali in grande delle curve chiuse sghembe, Rend. Mat. (6) 1 (1968) 237-297.

[5] J. L. Weiner, A theorem on closed space curves, Rend. Mat. (3) 8 (1975) 789-804. 